\title{
Simplified Shape Factors for a Thermal Diffusion Column
}

\author{
C. J. G. SLIEKer \\ F.O.M. - Laboratorium voor Massascheiding, Kruislaan 407, Amsterdam-O, Netherlands \\ (Z. Naturforsch?. 20 a, 521-526 [1965] ; received 15 July 1964)
}

\begin{abstract}
Starting from Cohes's theory of square cascades and countercurrent separation processes, column constants for the three types of thermal diffusion columns are calculated. It is assumed that the transport coefficients may be regarded as constants, whereas their values are taken at the mean temperature in the column. By comparison with other theories and experimental results our simplified theory appears to be as good as the more refined ones.
\end{abstract}

During the last years several authors have tried to perfect the theory of a thermal diffusion column by taking into account the temperature dependence of the transport coefficients. They have calculated the column constants for different molecular models ${ }^{1-3}$. But as a consequence of the complicated numerical calculations nearly all authors restrict themselves to the hot wire type, even though electronic computers are used. The column parameters are given as a function of the two independent parameters $T_{\mathrm{h}}$ and $r_{\mathrm{h}} / r_{\mathrm{c}}$ in the form of tables.

Now it appears that the calculated separation factor in the column is nearly independent of the choice of the molecular model ${ }^{4}$, so that the discrepancies between theory and experiment seem to be the results of imperfections in the general theory of countercurrent separation columns. Therefore, we have calculated in a simplified way the column constants, which give as good results as the more refined theories. We started from the general cascade theory of Conen in order to show that the column parameters come out in a natural and elegant way for al types of thermal diffusion columns.

\section{General Theory}

The behaviour of a binary mixture in a square cascade or countercurrent separation column is completely determined by four so-called square cascade or column constants $c_{1}, c_{2}, c_{3}$ and $c_{6}$. These

1 S. C. Saxena and S. Raman, J. Chem. Phys. 36, 3345 [1962].

2 B. B. McInteer and M. J. Reisfeld, J. Chem. Phys. 33, 570 [1960].

3 B. B. McInteer and M. J. Reisfeld, Los Alamos Scientific Laboratory report LAMS 2517. constants describe respectively the initial transport in the column $\left(c_{1}\right)$; the loss in separation, caused by back diffusion in vertical direction $\left(c_{2}\right)$; the losses due to the dragging effect of the countercurrent flow $\left(c_{3}\right)$; and the amount of material per unit length, the hold-up $c_{6}$. The enrichment of the component with mole fraction $N$ as a function of the time $t$, of the vertical coordinate $z$, and of the rate of withdrawal $P$ is given by the following expression ${ }^{5}$

$c_{6} \frac{\partial N}{\partial t}=c_{5} \frac{\partial^{2} N}{\partial z^{2}}-\frac{\partial}{\partial z}\left[P N+c_{1} N(1-N)\right]$.

$c_{5}$ describes the total loss: $c_{5}=c_{2}+c_{3}$. To this constant may be added another term, designated $c_{7}$ (in the notation of Furry and Jones ${ }^{6} K_{\mathrm{p}}$ ), which takes into account the losses in the separation as a consequence of improper column construction e. g. for thermal diffusion, irregularities in the temperature distribution and fluctuations in the distance between the hot and the cold wall. $P$ is the production rate of the column. The column constants can be written down in cartesian or cylindrical coordinates, according to the shape of the column.

In the cartesian case the column consists of two vertical plates with a width of $B \mathrm{~cm}$, a distance $d$ apart; the horizontal coordinate is denoted by $x$.

In cylindrical coordinates the column consists of two concentric cylinders, the inner with radius $r_{\mathrm{h}}$, the outer with radius $r_{\mathrm{c}}$. The horizontal coordinate is here represented by $r$.

${ }^{4}$ S. C. Saxena and S. Raman, Rev. Mod. Phys. 34, 252 [1962].

5 K. Conen, The Theory of Isotope Separation, McGraw-Hill Book Co., New York 1951.

6 R. C. Jones and W. H. Furry, Rev. Mod. Phys. 18, 151 [1946]. 
Cartesian coordinates

$c_{1}=-B \int_{0}^{d}(2 \alpha)_{0} \mathrm{~d} x \int_{0}^{x} \varrho v \mathrm{~d} x$,

$c_{2}=B \int_{0}^{d} \varrho D \mathrm{~d} x$,

$c_{3}=B \int_{0}^{d} \frac{\mathrm{d} x}{\varrho D}\left|\int_{0}^{x} \varrho v \mathrm{~d} x\right|^{2}$,

$c_{5}=c_{2}+c_{3}$,

$c_{6}=B \int_{0}^{d} \varrho \mathrm{d} x$,

$P=B \int_{0}^{d} \varrho v \mathrm{~d} x$,

$Q$ is the total density in the column in $\mathrm{g} / \mathrm{cm}^{3}$ and $D$ the diffusion coefficient of the binary mixture under consideration. $\left(2 \alpha_{0}\right)$ characterizes the separation process, e. g. ultra centrifuge, mass diffusion, chemical exchange, distillation or thermal diffusion. etc. For the latter:

$$
(2 \alpha)_{0}=\alpha \frac{\partial \ln T}{\partial x} \text { or } \quad(2 \alpha)_{0}=\alpha \frac{\partial \ln T}{\partial r}
$$

where $\alpha$ is the thermal diffusion factor and $T$ the absolute temperature $\left({ }^{\circ} \mathrm{K}\right)$.

The function $v$ in $c_{1}, c_{3}$ and $P$ describes the velocity of the countercurrent flow. This flow is either completely determined by the conditions of the elementary separation process and the shape of the column (e. g. thermal diffusion) or it can be forced upon the elementary separation and thus be used as an operating variable (e. g. ultra centrifuge and mass diffusion).

The solution of equation (1) has been given generally in terms of the column constants ${ }^{5}$ or it has been given in connection with the solution of specific separation processes ${ }^{6,7}$. But as a consequence of the different notations the agreement between the theories of these separation processes is not very lucid.

We will give a short review of the most important expressions, used in column operations in terms of the cascade constants (for a complete discussion see $\left.^{7,8}\right)$.

7 Proc. 2nd U. N. Intern. Conf. Peaceful Uses of Atomic Energy, Geneva 1958, Vol. 4, p. 608.

8 R. Fleischmann and H. Jensen, Ergebn. Exakt. Naturw. 20, 121 [1942].
Cylindrical coordinates

$$
\begin{aligned}
& c_{1}=-2 \pi \int_{r_{\mathrm{h}}}^{r_{\mathrm{c}}}(2 \alpha)_{0} \int_{r_{\mathrm{h}}}^{r} \varrho v r \mathrm{~d} r \mathrm{~g} / \mathrm{sec} ; \\
& c_{2}=2 \pi \int_{r_{\mathrm{h}}}^{r_{\mathrm{c}}} \varrho D r \mathrm{~d} r \quad \mathrm{~g} \mathrm{~cm} / \mathrm{sec} ; \\
& c_{3}=2 \pi \int_{r_{\mathrm{h}}}^{r_{\mathrm{c}}} \frac{\mathrm{d} r}{\varrho D r}\left[\int_{r_{\mathrm{h}}}^{r} \varrho v r \mathrm{~d} r\right]^{2} \mathrm{~g} \mathrm{~cm} / \mathrm{sec} ; \\
& c_{5}=c_{2}+c_{3} ; \\
& c_{6}=2 \pi \int_{r_{\mathrm{h}}}^{r_{\mathrm{c}}} \varrho r \mathrm{~d} r \quad \mathrm{~g} / \mathrm{cm} ; \\
& P=2 \pi \int_{r_{\mathrm{h}}}^{r_{\mathrm{c}}} \varrho v r \mathrm{~d} r \quad \mathrm{~g} / \mathrm{sec} .
\end{aligned}
$$

A. The separation factor in the stationnary state of a countercurrent column is given by

$$
\ln q=2 \varepsilon_{0} Z \frac{2 m}{1+m^{2}}
$$

with

$$
q \equiv \frac{[N / 1-N]_{\text {top }}}{[N / 1-N]_{\text {bottom }}},
$$

$Z$, the length of the column,

$2 \varepsilon_{0}=\frac{1}{2} c_{1} / \sqrt{c_{2} c_{3}}$ gives the maximum separation factor per $\mathrm{cm}$ length, which is still a function of the flow pattern,

$m=\sqrt{c_{3}} / \overline{c_{2}}$; the factor $2 m /\left(1+m^{2}\right)$ gives the contribution of the magnitude of flow to the separation factor.

The flow pattern in a thermal diffusion column is completely determined by the temperature difference of the hot and the cold wall and by the shape of the column; $2 \varepsilon_{0}$ has thus a fixed value in a thermal diffusion column.

B. The separative power ${ }^{9} \delta U$, a very important function in countercurrent devices with a certain production rate. For short columns the separative power is given by

$$
\delta U=\frac{c_{1}^{2}}{4 c_{3}} \frac{m^{2}}{1+m^{2}},
$$

$m^{2} /\left(1+m^{2}\right)$ gives the contribution of the magnitude of the flow to the separative power.

The optimum value of $m$ for producing separated isotopes, or for getting a separation is totally dif-

\footnotetext{
9 The separative power $\delta U$ is called maximum differential separative power $\mathrm{d} U_{\max } / \mathrm{d} z$ by H. London (Separation of Isotopes, London 1961), and a measure of effectiveness of a unit length $H^{2} / 4 K$ by Jones and F UrrY ${ }^{6}$.
} 
ferent, because

$$
\begin{aligned}
& \delta U=(\delta U)_{\max } \text { for } m \rightarrow \infty, \\
& \ln q=(\ln q)_{\max } \quad \text { for } \quad m=1 .
\end{aligned}
$$

The approach to $(\delta U)_{\max }$, however, is rapid, as can be seen from Fig. 1, e. g. at $m=3$,

$$
\delta U=0.90(\delta U)_{\max }, \quad \ln q=0.60(\ln q)_{\max } .
$$

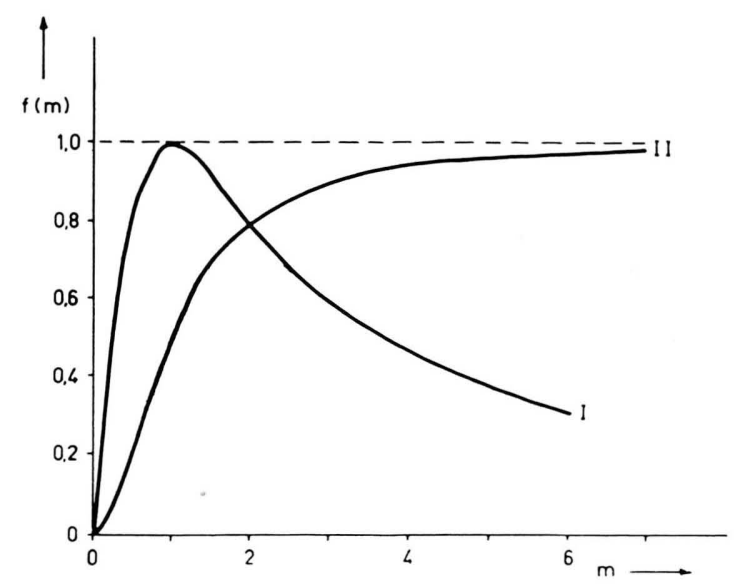

Fig. 1. The separation factor and separative power as a function of the magnitude of flow.

I: $f(m)=q / q_{\max }=2 m /\left(1+m^{2}\right)$,

II : $f(m)=\delta u / \delta u_{\max }=m^{2} /\left(1+m^{2}\right)$.

For the description of the behaviour of a thermal diffusion column it is only necessary to evaluate the column constants $c_{1}, c_{2}, c_{3}, c_{6}$ and $P$ for different shapes of the column. The most important cases are:

1. plane parallel plates,

2. concentric cylinders,

3 . hot wire.

Flat columns strictly only exist in theory as it is nearly impossible to construct them properly. Its theory can be used, however, in first approximation for the concentric cylinder type, provided that the annular gap is small and the radii of the cylinders large. The hot wire column is obtained from the concentric cylinder type by taking $r_{\mathrm{h}} / r_{\mathrm{c}} \ll 1$.

The most difficult problem in calculating the behaviour of the column arises from the fact that the constants $c_{1}, c_{2}$ and $c_{3}$ contain the transport coefficients $\lambda$ (thermal conductivity); $\eta$ (the viscosity); $\alpha$ and $D$. It is thus necessary to introduce molecular interaction models, with the help of which the temperature dependence of the transport coefficients can be determined. During the last years several authors have calculated the column constants for different molecular models:

1. the rigid sphere model ${ }^{1}$,

2. the inverse power model ${ }^{3}$,

3. the LenNard-Jones model ${ }^{2}$.

It seems, however, that these authors did not consider that the discrepancies between theory and experiment are not only caused by the incomplete knowledge of the column constants, but also by imperfections in the general theory of the separation column.

We have made the assumption that the transport coefficients may be regarded as constants in first approximation, taking their values at the mean temperature $\bar{T}$ in the columns.

\section{The Plane Case}

This type of column consists of two vertical parallel surfaces, a distance $d$ apart. The width of the plates is $B \mathrm{~cm}$. The temperatures of the surfaces are $T_{\mathrm{h}}$ and $T_{\mathrm{c}} ; \Delta T=T_{\mathrm{h}}-T_{\mathrm{c}} ; \quad T=\frac{1}{2}\left(T_{\mathrm{h}}+T_{\mathrm{c}}\right)$.

The function $v$, which specifies the flow can be obtained by solving the Navier-Stokes equation with suitable boundary conditions. The procedure has been extensively described elsewhere ${ }^{7,8}$. The following equation describes the vertical flow as a function of $x$.

$$
v=-\frac{1}{12} \frac{\varrho g}{\eta} \frac{\Delta T}{T} \frac{x}{d}(2 x-d)(x-d)
$$

where $g$ is the acceleration of gravity.

The column constants become

$$
\begin{aligned}
& c_{1}=\frac{d^{3} \varrho^{2} a g}{6 ! \eta} B\left\{\frac{\Delta T}{T}\right\}^{2}, \\
& c_{2}=\varrho D d B \\
& c_{3}=\frac{d^{7} \varrho^{3} g^{2}}{9 ! \eta^{2} D} B\left\{\frac{\Delta T}{T}\right\}^{2},
\end{aligned}
$$

results, which coincide with the column constants calculated by FurRy and Jones ${ }^{7}$

$$
c_{1}=H^{(0)}, \quad c_{2}=K_{\mathrm{d}}^{(0)}, \quad c_{3}=K_{\mathrm{c}}^{(0)} .
$$

Furthermore

where

$$
\begin{aligned}
& \ln q=\frac{Z}{2,4 d} \alpha \frac{\Delta T}{T} \frac{2 m}{1+m^{2}} \\
& m=\sqrt{\frac{c_{3}}{c_{2}}}=\frac{\varrho g d^{3}}{602 \eta D} \frac{\Delta T}{T} .
\end{aligned}
$$

Assuming that $\varrho \sim p$ and $D \sim p^{-1}$ we get $m \sim p^{2}$. Bearing in mind that $q$ has its maximum value at 
$m=1$, we can define an optimal pressure $p_{\text {opt }}$ :

$$
p_{\text {opt }}^{2}=602 p_{0}^{2} \underset{\varrho_{0} g d^{3} \Delta T}{\varrho^{3} \Delta T}
$$

and thus

$$
m=\left\{p / p_{\text {opt }}\right\}^{2},
$$

where $p_{0}$ is some reference pressure, where $Q=\varrho_{0}$ and $D=D_{0} ; p_{0}$ is usually taken $76 \mathrm{~cm} \mathrm{Hg}$.

From (13) the spacing at the optimal pressure, $d\left(p_{\text {opt }}\right)$ is obtained

$$
d\left(p_{\text {opt }}\right)=8.44 \sqrt[3]{\frac{\eta D T}{\varrho g \Delta T}}
$$

but this is not the optimal spacing $d_{\text {opt }}$ with regard to the separation factor.

Differentiation of (12) with respect to $d$ yields the latter:

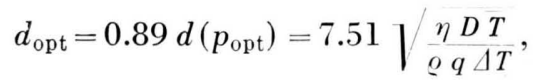

This is an important fact to remember when designing columns. The separative power is given by

$$
\delta U=\frac{0.175 Z}{d} \varrho D B\left\{\alpha \frac{\Delta T}{T}\right\}^{2} \frac{m^{2}}{1+m^{2}}
$$

and the optimum spacing with regard to the separative power becomes

$$
d_{\text {opt }}^{*}=1.3 d\left(p_{\text {opt }}\right) .
$$

This fact was already known experimentally. As a rule of thumb the diameter of a column for production is chosen $1.2-1.3$ times the diameter at

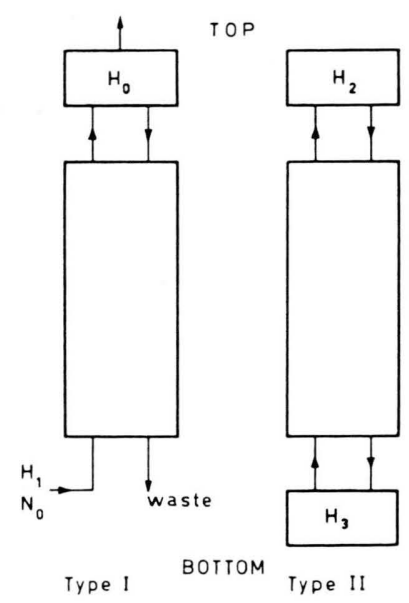

Fig. 2. The two principal types of column operation. zero production. Furthermore, it can be seen from (18) that thermal diffusion must be carried out in the gaseous state, as the product $\varrho D$ is here $10^{2}$ times larger than in the liquid state.

Most of the formulas presented in this paragraph are already given by other authors $7,8,10$, we only wanted to show that the expressions can be derived from Conen's cascade theory in a very simple way.

\section{The Concentric Cylinder Case}

The Navier-Stokes equation in cylindrical coordinates becomes

$$
\begin{aligned}
& \frac{\partial^{3} v}{\partial r^{3}}+\frac{1}{r} \frac{\partial^{2} v}{\partial r^{2}}-\frac{1}{r^{2}} \frac{\partial v}{\partial r}=\frac{\rho g}{\eta T} \frac{\partial T}{\partial r} \\
& \frac{\partial T}{\partial r}=\frac{1}{r} \frac{\Delta T}{\ln \left(r_{\mathrm{h}} / r_{\mathrm{c}}\right)}, \quad \text { see ref. }{ }^{8} .
\end{aligned}
$$

Equation (20) is easily solved by putting $r=e^{x}$, giving:

$v=A_{1}+A_{2} \ln r+A_{3} r^{2}+\frac{1}{4}\left\{\begin{array}{ccc}\varrho g & \mid T & 1 \\ \eta & T & \ln a\end{array}\right\} r^{2} \ln r$

with

$$
a=r_{\mathrm{h}} / r_{\mathrm{c}} .
$$

The integration constants $A_{1}, A_{2}$ and $A_{3}$ are determined by the boundary conditions:

$$
v=0, \quad r=r_{\mathrm{c}} ; \quad v=0, \quad r=r_{\mathrm{h}},
$$

$\int_{i_{\mathrm{h}}}^{r_{\mathrm{c}}} \varrho v r \mathrm{~d} r=0$, no transport in vertical direction,

$$
P=0 \text {. }
$$

The calculation of $A_{1}, A_{2}$ and $A_{3}$ is simplified by introducing the new variable

$$
p=r / r_{\mathrm{c}}
$$

which changes (22) into

$$
\begin{aligned}
& v=G r_{\mathrm{c}}^{2}\left\{K_{1}+K_{2} \ln \varphi+K_{3} \varphi^{2}+\frac{1}{4} \varphi^{2} \ln \varphi\right\} \\
& \text { with } \quad G=\frac{\varrho g}{\eta} \frac{\Delta T}{T} \frac{1}{\ln a} \\
& \text { and } \quad K_{1}=\frac{A_{1}}{G r_{\mathrm{c}}{ }^{2}}+\frac{A_{2}}{G r_{\mathrm{c}}{ }^{2}} \ln r_{\mathrm{c}}, \\
& K_{2}=A_{2} /\left(G r_{\mathrm{c}}{ }^{2}\right) \text {, } \\
& K_{3}=\frac{1}{4} \ln r_{\mathrm{c}}+A_{3} / G .
\end{aligned}
$$

Calculation of $K_{1}, K_{2}$ and $K_{3}$ from (23) yields:

$$
K_{1}=-K_{3}=\frac{1}{4\left(1-a^{2}\right)}\left\{a^{2} \ln a-\frac{1}{4} \ln a \frac{\left(1-a^{4}\right)+4 a^{2} \ln \mathrm{a}}{\left(1+a^{2}\right) \ln a+\left(1-a^{2}\right)}\right\},
$$

10 L. Waldmann, Z. Phys. 114, 53 [1939]. 


$$
K_{2}=-\begin{array}{cc}
1 & 1-a^{4}+4 a^{2} \ln \mathrm{a} \\
16 & \left(1+a^{2}\right) \ln a+\left(1-a^{2}\right)
\end{array} .
$$

With

$$
T=T_{\mathrm{c}}-\Delta T(\ln \varphi / \ln a)
$$

and $\frac{\partial \ln T}{\partial r}=r_{\mathrm{c}}{ }^{\partial \ln T} \partial r_{\mathrm{c}} \frac{1}{T} \frac{\partial T}{\partial \varphi}\left(\right.$ ref. $\left.^{8}\right)$

the column constants can be calculated, according to $(2)$.

The mean temperature in a concentric cylinder and hot wire column is approximately given by the formula of Fleischmann and Jensen ${ }^{8}$

$$
\bar{T}=T_{\mathrm{c}}-\Delta T(0.56 / \ln a) .
$$

The parameters $c_{1}$ and $c_{3}$ can be given explicitly, but as their geometry constants are rather complex, they will be written in the form

$$
\begin{gathered}
c_{1}=[\mathrm{S} . \mathrm{F} .]_{1} r_{\mathrm{c}} \frac{\varrho^{2} g \alpha}{\eta}\left\{\frac{\Delta T}{T}\right\}^{2}, \\
c_{3}=[\mathrm{S} . \mathrm{F} .]_{3} r_{\mathrm{c}}{ }^{8} \frac{\varrho^{3} g^{2}}{\eta^{2} D}\left\{\frac{\Delta T}{T}\right\}^{2}
\end{gathered}
$$

where $[\mathrm{S} . \mathrm{F} .]_{1}$ and $[\mathrm{S} . \mathrm{F} .]_{3}$ are shape factors, which are calculated numerically on an I.B.M. 1620 computer. They are tabulated in Table 1.

The calculation of $c_{2}$ gives no difficulties

$$
c_{2}=\pi g D r_{\mathrm{c}}^{2}\left(1-a^{2}\right) .
$$

\begin{tabular}{|l|c|c|}
\hline$a^{-1}$ & {$[\mathrm{~S} . \mathrm{F} .]_{1} 6 !$} & {$[\mathrm{S} . \mathrm{F} .]_{3} 9 !$} \\
\hline 1.2 & 0.0259 & $0.1956 \cdot 10^{-4}$ \\
1.5 & 0.1885 & $0.2302 \cdot 10^{-2}$ \\
2 & 0.5433 & 0.0334 \\
2.5 & 0.8274 & 0.1042 \\
3 & 1.0220 & 0.1814 \\
3.5 & 1.1497 & 0.2855 \\
4 & 1.2317 & 0.3682 \\
4.3 & 1.2654 & 0.4140 \\
4.5 & 1.2830 & 0.4399 \\
5 & 1.3137 & 0.5017 \\
6 & 1.3375 & 0.5971 \\
7 & 1.3338 & 0.6607 \\
8 & 1.3169 & 0.7037 \\
9 & 1.2939 & 0.7313 \\
10 & 1.2683 & 0.7526 \\
11 & 1.2420 & 0.7658 \\
12 & 1.2160 & 0.7703 \\
13 & 1.1908 & 0.7774 \\
14 & 1.1667 & 0.7747 \\
15 & 1.1437 & 0.7808 \\
20 & 1.0458 & 0.7617 \\
25 & 0.9709 & 0.7374 \\
30 & 0.9121 & 0.7088 \\
35 & 0.8646 & 0.6961 \\
40 & 0.8252 & 0.6698 \\
50 & 0.7635 & 0.6348 \\
\hline
\end{tabular}

Table 1. Shape factors for a concentric cylinder column.

\section{The Hot Wire Type}

Strictly speaking, it is not necessary to treat this type of column in a separate paragraph, as all the expressions required are derived in the previous section. The calculations are simplified, however, by neglecting all factors

$\left.\mid \begin{array}{l}r_{\mathrm{h}} \\ r_{\mathrm{c}}\end{array}\right\}^{n}$ and $4\left\{\left\{\frac{r_{\mathrm{h}}}{r_{\mathrm{c}}}\right\}^{n} \ln { }_{r_{\mathrm{c}}}^{r_{\mathrm{h}}}\right.$ for $n \geqq 2$ with respect to 1 .

The resulting expressions are

$$
\begin{gathered}
K_{2}=-\frac{1}{16} \frac{1}{1+\ln a} \\
K_{3}=\frac{1}{4} a^{2} \ln a-\frac{1}{16} \frac{\ln a}{1+\ln a}
\end{gathered}
$$

and thus

$$
\begin{aligned}
& c_{1}=\frac{\pi}{2^{7}} \frac{2+\ln a}{(\ln a)^{2}(1+\ln a)} \cdot \stackrel{Q^{2} g \alpha}{\eta} r_{\mathrm{c}}{ }^{4}\left\{\frac{\Delta T}{T}\right\}^{2}, \\
& c_{2}=\pi \varrho D r_{\mathrm{c}}^{2} \text {, } \\
& c_{3}=\underset{3^{3} \cdot 2^{15}}{\pi} \frac{\left[15(\ln a)^{2}+56 \ln a+53\right]}{(1+\ln a)^{2}(\ln a)^{2}} \cdot r_{\mathrm{c}}{ }^{8} \frac{\varrho^{3} g^{2}}{\eta^{2} D}\left\{\frac{\Delta T}{T}\right\}^{2} .
\end{aligned}
$$

\begin{tabular}{|c|c|c|}
\hline$a^{-1}$ & {$[\text { S.F. }]_{1} 6 !$} & {$[\text { S.F. }]_{3} 9 !$} \\
\hline 15 & 0.999 & 0.683 \\
20 & 0.982 & 0.716 \\
25 & 0.937 & 0.711 \\
30 & 0.891 & 0.696 \\
35 & 0.851 & 0.679 \\
40 & 0.816 & 0.661 \\
50 & 0.758 & 0.630 \\
\hline
\end{tabular}

Table 2. Shape factors for a hot wire column.

The geometry constants of (40) and (41) are calculated and given in Table 2. Comparison of the results of Table 1 and Table 2 show that the simplified expressions for the hot wire type may be used starting from $a^{-1}=25$.

\section{Comparison of Theory and Experiment}

To check the utility of our calculations a comparison is made with the theoretical results, reviewed by Saxena and Raman ${ }^{4}$ and with the experimental work of McInteer, Aldrich, and Nier ${ }^{11}$. The latter separate the helium isotopes in a concentric cylinder and a hot wire column and give the experimental values of $c_{1}$ and $2 \varepsilon=c_{1} /\left(c_{2}+c_{3}\right)$.

11 B. B. McInteer, L. T. Aldrich, and A. O. C. Nier, Phys. Rev. 74, 946 [1948]. 
The dimensions and transport properties (calculated with the first approximation of the ChapmanEnskog theory for a Lennard-Jones potential) of McInteer's columns are listed in Tables 3 and 4 .

\begin{tabular}{|l|ll|}
\hline$r_{\mathrm{c}}=0.466 \mathrm{~cm}$ & $\alpha=0.059$ & \\
$r_{\mathrm{h}}=0.018 \mathrm{~cm}$ & $\varrho_{300}=16.3 \cdot 10^{-5} \mathrm{~g} / \mathrm{cm}^{3}(1 \mathrm{~atm}), Q \sim T^{-1}$ \\
$T_{\mathrm{c}}=300^{\circ} \mathrm{K}$ & $\eta_{300}=200 \cdot 10^{-6} \mathrm{~g} / \mathrm{cm} \mathrm{sec}^{\circ}$, & $\eta \sim T^{1 / 2}$ \\
$T_{\mathrm{h}}=1100^{\circ} \mathrm{K}$ & $D_{300}=1.66 \mathrm{~cm}^{2} / \mathrm{sec}(1 \mathrm{~atm})$, & $D \sim T^{3 / 2}$
\end{tabular}

Table 3. Characteristics of McInteER's hot wire column.

$$
\begin{array}{l|l}
r_{\mathrm{c}}=3.65 \mathrm{~cm} & T_{\mathrm{c}}=300{ }^{\circ} \mathrm{K} \\
r_{\mathrm{h}}=3.02 \mathrm{~cm} & T_{\mathrm{h}}=600^{\circ} \mathrm{K} \quad T=450{ }^{\circ} \mathrm{K}
\end{array}
$$

Table 4. Characteristics of McInteER's concentric cylinder column.

We see from Table 5 that our theory gives quite as good results as the more refined ones; the agreement between theory and experiment remains poor, but an improvement of this agreement should not be expected from these simple calculations: the advantage is that one could readily calculate with reasonable accuracy the influence of the independent variables $a$ and $T$ on the efficiency of the column.

It is a pleasure to acknowledge Dr. J. Los and Dr. A. E. DE VRIEs for their most helpful discussions.

This work is part of the research program of the Stichting voor Fundamental Onderzoek der Materie and was made possible by financial support from the Neder- landse Organisatie voor Zuiver-Wetenschappelijk Onderzoek.

\begin{tabular}{|l|c|cc|}
\hline & Hot wire & \multicolumn{2}{c|}{$\begin{array}{c}\text { Conc. cylinder } \\
\text { column }\end{array}$} \\
\hline \multicolumn{1}{|c|}{ pressure (atm) } & 7.8 & 9.7 & 7.8 \\
\hline$c_{1}$ (exptl) litres STP/Day & 16.5 & 24.8 & 257 \\
$c_{1}$ (a) & 9.5 & 14.8 & 267 \\
$c_{1}$ (b) & 12.5 & 19.3 & - \\
$c_{1}$ (c) & 12.1 & 18.8 & 282 \\
$c_{1}$ (present theory) & 20.0 & 30.8 & 238 \\
\hline$c_{2}$ (exptl) $\mathrm{cm}^{3} \mathrm{STP} / \mathrm{cm}$ sec & & & \\
$c_{2}$ (a) & 1.70 & 1.70 & 26.7 \\
$c_{2}$ (b) & 1.25 & 1.25 & - \\
$c_{2}$ (c) & 1.36 & 1.36 & 26.1 \\
$c_{2}$ (present theory) & 1.24 & 1.24 & 28.7 \\
\hline$c_{3}$ (exptl) $\mathrm{cm}^{3} \mathrm{STP} / \mathrm{cm} \mathrm{sec}$ & & & \\
$c_{3}$ (a) & - & - & - \\
$c_{3}$ (b) & 0.443 & 1.06 & 129 \\
$c_{3}$ (c) & 1.382 & 3.31 & - \\
$c_{3}$ (present theory) & 0.940 & 2.24 & 134 \\
\hline $2 \varepsilon$ (exptl) $\mathrm{cm}^{-1}$ & 2.94 & 6.97 & 106 \\
$2 \varepsilon$ (a) & & & \\
$2 \varepsilon$ (b) & 0.043 & - & 0.017 \\
$2 \varepsilon$ (c) & 0.051 & 0.062 & 0.020 \\
$2 \varepsilon$ (present theory) & 0.055 & 0.032 & - \\
\hline
\end{tabular}

Table 5. Experimental and calculated values of the column constants $c_{1}, c_{2}, c_{3}$ and $2 \varepsilon$.

(a) inverse power model, Maxwellian molecules

(b) rigid spherical molecules

(c) molecules with a LenNaRd-Jones potential. 